\title{
Las transformaciones del papel económico de los municipios y la necesidad de cambios en la regulación de la intervención económica local
}

\author{
Ricardo Rivero Ortega \\ Profesor Titular de Derecho Administrativo \\ Universidad de Salamanca
}

SUMARIO: I. INTRODUCCIÓN. II. EL TRADICIONAL PAPEL MUNICIPAL DE PRESTACIÓN DE SERVICIOS HASTA LA LEY DE BASES EN RÉGIMEN LOCAL DE 1985. III. LA CRISIS DEL INTERVENCIONISMO DIRECTO EN LOS AÑOS OCHENTA Y NOVENTA. IV. ¿CÓMO PUEDEN DESARROLLAR HOY SU INTERVENCIÓN ECONÓMICA LOS ENTES LOCALES?: LA NECESIDAD DE UNA ACTUALIZACIÓN NORMATIVA Y EL PROYECTO DE LEY DE MODERNIZACIÓN DE LA ADMINISTRACIÓN LOCAL. V. LA ACTIVIDAD ECONÓMICA DE LOS ENTES LOCALES EN COLABORACIÓN CON OTROS ENTES PÚBLICOS, LA COLABORACIÓN PÚBLICO-PRIVADO, Y LA REGULACIÓN DE LA OPCIÓN INSTITUCIONAL.

\section{INTRODUCCIÓN}

Este breve estudio, modesto homenaje a uno de los grandes maestros de nuestro Derecho administrativo, y gran especialista en Derecho administrativo económico ${ }^{1}$, pretende avanzar algunas reflexiones sobre la transformación experimentada en el papel intervencionista de las Corporaciones locales sobre la economía, transformación que debiera tal vez producir consecuencias normativas. En el modelo europeo de desarrollo los entes locales han representado tradicionalmente un papel decisivo, aunque durante las últimas décadas han tenido que ceder gran parte de su protagonismo, especialmente en nuestro país, debido a la aparición de los nuevos poderes administrativos de las Comunidades Autónomas. El fenómeno de sustracción de competencias municipales (también provinciales) por los poderes autonómicos, unido a la tendencia internacional de privatización y liberalización de servicios, ha dejado a los entes locales - ésta sería la primera tesis del estudio- menos capacitados para ejercer la iniciativa económica constitucionalmente reconocida empleando exclusivamente sus propios medios.

\footnotetext{
${ }^{1}$ Vid. Sebastián Martín-Retortillo, Derecho Administrativo Económico, vol. I, La Ley, Madrid, 1988.
} 
Ante esta situación, más o menos acentuada dependiendo de la estructura municipal y provincial de cada Comunidad Autónoma, y de sus circunstancias económicas, se hace preciso replantear la intervención de los entes locales en pro del desarrollo. Para ello se requiere — segunda tesis de este trabajo - una nueva regulación de las técnicas de intervención de los entes locales, respetuosa con el principio constitucional de autonomía y al mismo tiempo garantizadora de un instrumental en manos de municipios y diputaciones que les permita satisfacer las necesidades de actuación sobre la economía que se les plantean. Esta regulación, preferiblemente enmarcable en la Ley de Bases de Régimen Local, en el capítulo sobre «Actividades y Servicios» (artículos 84 a 87), podría contemplar un catálogo más completo, y sobre todo adaptado a las nuevas circunstancias económicas y de reparto de competencias, de las opciones institucionales básicas para la realización de la intervención económica de las Corporaciones locales.

\section{EL TRADICIONAL PAPEL MUNICIPAL DE PRESTACIÓN DE SERVICIOS HASTA LA LEY DE BASES DE RÉGIMEN LOCAL DE 1985}

Es un lugar común recordar que desde la Edad Media los municipios se han preocupado especialmente de garantizar a sus vecinos unas condiciones vitales mínimas ${ }^{2}$, previniendo situaciones de escasez y estimulando la realización de actividades necesarias para mejorar su calidad de vida ${ }^{3}$. Su papel sobre los mercados no puede dejar de recordarse, máxime si tenemos en cuenta que hasta hoy la cuestión de los abastos les ha seguido incumbiendo. La organización de pósitos, los mataderos municipales y la recogida de basuras, por citar sólo tres ejemplos, demuestran a lo largo de la historia la importancia de la organización de los poderes locales para el bienestar de los ciudadanos ${ }^{4}$. Con este fin se han desarrollado múltiples formas de gestion, objeto de análisis y comentario por la literatura jurídico-administrativa, desde el clásico ALBI ${ }^{5}$ hasta la referencia obligada hoy de Sosa Wagner ${ }^{6}$. Los buenos conocedores de la historia institucional

\footnotetext{
${ }^{2}$ Vid. Cirilo Martín-Retortillo, Las obligaciones mínimas de los Ayuntamientos, Reus, Madrid, 1956.

${ }^{3}$ Vid. García de Enterría, «La actividad industrial y mercantil de los municipios», Revista de Administración Pública núm.17, Mayo-Agosto 1955.

${ }^{4}$ Vid. Clavero Arévalo, Municipalización y provincialización de servicios en la Ley de Régimen Local, Madrid, IEAL, 1952.

${ }^{5}$ Vid. Albi, Tratado de los modos de gestión de las Corporaciones locales, Madrid, 1960.

${ }^{6}$ Vid. Sosa Wagner, La gestión de los servicios públicos locales, Civitas, Madrid, 2002.
} 
europea saben que mucho antes de la construcción del Estado de Bienestar se fue trabando una red de municipios empeñados en mejorar la situación de sus vecinos.

A lo largo del siglo xx el protagonismo municipal se va incrementando como consecuencia de las tendencias demográficas determinadas por el éxodo rural. La acumulación de grandes masas de población en las principales ciudades obliga a los gobiernos municipales a garantizar nuevos servicios para evitar que el hacinamiento y las condiciones higiénicas terminen por perjudicar a todos los habitantes. Entonces los Ayuntamientos se preocuparán de que no falte lo más básico, comenzando por el pan, sujeto a intensas reglamentaciones de calidad y precio ${ }^{7}$, y el agua, con el establecimiento de los primeros servicios de abastecimiento domiciliario a poblaciones ${ }^{8}$. Después de las guerras mundiales, muy especialmente, crecen también las dimensiones organizativas de los entes locales por mor de la creación de numerosas empresas públicas dirigidas precisamente a prestar, en condiciones más flexibles que las propias de los entes administrativos, los servicios de contenido económico.

En nuestro país, el proceso será más lento y tardío que en otras naciones europeas (Alemania, Inglaterra, Francia), pioneras de la revolución industrial, pero en el primer cuarto de la pasada centuria ya se aprobará el Estatuto Calvo Sotelo, una norma diseñada para responder a las nuevas exigencias sociales y económicas. Las normas posteriores sobre régimen local profundizaron en esta línea, como puede observarse en la Ley de Régimen local de 1955. El Reglamento de Servicios de las Corporaciones locales de 1955, no menos relevante que las anteriores leyes, supondrá la consagración normativa de estas tendencias, al regular pormenorizadamente las distintas herramientas en manos de la Administración local para desarrollar sus funciones económicas. La ausencia de verdadera descentralización, sin embargo, dificultaba que los municipios desarrollaran un papel relevante de fomento y estímulo de la economía ${ }^{9}$.

Tras la Constitución, la Ley de Bases de Régimen Local de 1985 (LBRL) recoge toda la tradición anterior de protagonismo de los municipios y la refuerza con la exigencia de prestación de los servicios mínimos

\footnotetext{
${ }^{7}$ Vid. ToRnos Mas, Régimen jurídico de la intervención de los poderes públicos en relación con los precios y tarifas, Bolonia, 1982.

${ }^{8}$ Vid. GARRIDO LOPERA, El servicio de abastecimiento de agua a poblaciones, Instituto de Estudios de Administración Local, Madrid, 1973.

${ }^{9}$ Vid. Manzanedo Mateos/Parada Vázquez, Corporaciones locales y desarrollo económico, Montecorvo, Madrid, 1971.
} 
obligatorios (artículo 26) y la declaración de reserva de numerosas actividades en favor de las entidades locales (artículo 86). La municipalización de servicios (el abastecimiento y la depuración de aguas; la recogida, tratamiento y aprovechamiento de los residuos; el suministro de gas y calefacción; los mataderos, mercados y lonjas centrales; el transporte público de viajeros; y los servicios mortuorios) demuestra la intención del legislador básico de entonces de consolidar la responsabilidad de los municipios por los servicios básicos, así como el correlativo derecho vecinal a reclamar su prestación ${ }^{10}$. Para las Diputaciones provinciales, también se mantiene un protagonismo en todo lo relacionado con la prestación de servicios, sobre todo en los pequeños municipios, y la realización de las tareas de fomento.

\section{LA CRISIS DEL INTERVENCIONISMO DIRECTO EN LOS AÑOS OCHENTA Y NOVENTA}

Paradójicamente, la cúspide del reconocimiento normativo del papel económico de los entes locales en la Ley de Bases de Régimen Local coincide con el auge de una tendencia internacional en pro de la privatización y liberalización de servicios tradicionalmente prestados por el sector público ${ }^{11}$. Las nuevas políticas económicas ensayadas en Gran Bretaña por el gobierno conservador de Margaret Thatcher servirán de punto de referencia para proponer toda una serie de reformas en el resto de los países europeos tendentes a rebajar los niveles de compromiso de los poderes públicos, incluyendo los municipales, en la satisfacción de las necesidades básicas de los ciudadanos. Estas doctrinas parecen haberse impuesto, pero debe advertirse que el objetivo de «adelgazamiento del Estado», inocuo y seguramente necesario si pensamos en las grandes estructuras administrativas estatales, se torna mucho más problemático al proyectarse sobre poderes locales considerablemente debilitados por la situación económica y la escasez de recursos para atender a numerosos intereses generales.

Las tendencias de desintervención se han traducido en privatizaciones y desaparición de los monopolios locales. En nuestro país, aunque también se han producido análisis de conjunto sobre la cuestión ${ }^{12}$, la mayor parte

\footnotetext{
${ }^{10}$ Vid. QuinTana LóPEZ, El derecho de los vecinos a la prestación y establecimiento de los servicios públicos obligatorios, Civitas, Madrid, 1987.

${ }^{11}$ Vid. Sebastián Martín-Retortillo, «Las empresas públicas: reflexiones del momento presente», Revista de Administración Pública núm. 126, 1991.
}

12 Vid. Díaz Lema, Los monopolios locales, Montecorvo, Madrid, 1993. 
de los estudios han girado en torno a algunos servicios, abogando por la retirada de la presencia pública directa, para permitir que el capital privado realice inversiones necesarias y favorecer la eficiencia mediante la introducción de la competencia con lo que supone de elección libre entre distintos proveedores de un mismo servicio. Los servicios funerarios ${ }^{13}$, el gas ${ }^{14}$ y el agua ${ }^{15}$ han representado campos de prueba del nuevo reparto de responsabilidades y papeles entre poder público e iniciativa privada. Las políticas de reforma estructural desde 1996 han seguido este criterio afectando a algunas actividades municipalizadas hasta el momento ${ }^{16}$.

No cabe duda de que algunos de los servicios tradicionalmente encomendados a los municipios tienen hoy mucho menos sentido, siendo menos necesarios que hace veinte, treinta o cuarenta años. Así sucede, por ejemplo, con el mercado, servicio exigible en municipios de más de 5.000 habitantes según el apartado b) del artículo 26 de la Ley de Bases de Régimen Local, traído a este precepto por la tradicional exigencia recogida en nuestra normativa anterior, pero sobrepasado por las nuevas circunstancias de creciente competencia entre grandes superficies, supermercados y todo tipo de establecimientos dedicados a la venta de productos alimenticios, verdaderos garantizadores de una oferta suficiente en todo municipio de determinadas dimensiones. La realidad, hay que decirlo, se ha mostrado tozuda frente a una regulación cristalizada justo en el momento en el que se invertía el proceso de consolidación del papel económico de los entes locales.

\section{IV. ¿CÓMO PUEDEN DESARROLLAR HOY SU INTERVENCIÓN ECONÓMICA LOS ENTES LOCALES?: LA NECESIDAD DE UNA ACTUALIZACIÓN NORMATIVA Y EL PROYECTO DE LEY DE MODERNIZACIÓN DE LA ADMINISTRACIÓN LOCAL}

A pesar de la retirada paulatina de los poderes municipales de algunos ámbitos de actividad que hasta el momento les correspondían, los servi-

\footnotetext{
13 Vid. GARCía DE CocA, Liberalización de la actividad funeraria, Universidad de Valladolid, 1998.

${ }^{14}$ Vid. Del Guayo, El servicio público del gas, Marcial Pons, 1997.

15 Vid. GonzÁlez-Antón, Los servicios públicos locales del agua: régimen jurídico de su gestión integrada, Universidad de León, 1999.

16 Vid. Díaz Lema, «La privatización en el ámbito local (el lento declive de los monopolios locales)», Revista de Estudios de la Administración Local y Autonómica núm. 282, Enero-Abril 2000.
} 
cios públicos obligatorios siguen existiendo, incluso en el contexto de una economía liberalizada y, a decir de algunos, también desregulada. No todo ha sido retirada y reconsideración de servicios, pudiendo señalarse también alguna ampliación de las obligaciones municipales, por ejemplo, en el ámbito de los residuos ${ }^{17}$. Pero es cierto que se ha ido reduciendo el margen de intervencionismo obligado de los entes locales para permitir una presencia mayor, y también más competitiva, de empresas privadas prestadoras de los servicios básicos. Se entiende que la iniciativa privada puede satisfacer las demandas sociales de calidad y suficiencia de estos servicios de forma más eficiente que como lo haría la propia Administración ${ }^{18}$.

No obstante las tendencias de reducción del papel económico de los entes locales, apenas ha cambiado en ese punto la normativa básica, manteniéndose todavía las potencialidades previstas en la cláusula general del 25.1 LBRL y la habilitación genérica para intervenir en la actividad económica del artículo 86 de la LBRL, en su primer apartado: «Las entidades locales, mediante expediente acreditativo de la conveniencia y oportunidad de la medida, podrán ejercer la iniciativa pública para el ejercicio de actividades económicas conforme al artículo 128.2 de la Constitución». Este precepto, asociado al texto constitucional, autoriza la creación de empresas públicas locales para desarrollar toda clase de actividades tendentes a «satisfacer las necesidades y aspiraciones de la comunidad vecinal». Aunque en distintos «grados», los municipios pueden aún realizar todo tipo de actividades de interés local, pues tienen capacidad reconocida para ello, asumen competencias en variados ámbitos de contenido económico, en los términos fijados por la legislación autonómica y estatal y, en fin, pueden reservarse incluso servicios, hasta en régimen de monopolio ${ }^{19}$.

Sin embargo, no puede desconocerse que las posibilidades efectivas de «reserva», especialmente en régimen de monopolio, y la iniciativa genérica para emprender todo tipo de actividades, se han visto limitadas como consecuencia del Derecho europeo sobre la intervención económica públi-

\footnotetext{
${ }^{17}$ Vid. Alenza García, El sistema de la gestión de residuos sólidos urbanos en el Derecho español, MAP, Madrid, 1997.

${ }^{18}$ Vid. GARCía de CoCA, «El servicio público local de abastecimiento de agua potable, saneamiento y depuración. Nuevas tendencias, en especial su gestion indirecta», en SOSA WAGNER (Coord.), El Derecho Administrativo en el umbral del siglo XXI, Homenaje al Profesor Dr. D. Ramón MARTín Mateo, Tirant lo Blanch, Valencia, 2000.

19 Vid. Fernández GonZÁLEZ, La intervención del municipio en la actividad económica, Civitas, Madrid, 1995
} 
ca, con proyecciones también sobre los entes locales ${ }^{20}$, así como por resoluciones judicales restrictivas de las capacidades de intervención de los municipios ${ }^{21}$ y si desde un punto de vista político pueden cuestionarse estas restricciones, no puede ocultarse en cambio que representan una evolución considerable en la manera de entender la actividad de los poderes locales en el terreno de la economía.

Las competencias económicas de los entes locales han resultado al mismo tiempo aminoradas como consecuencia de la entrada en el panorama administrativo de las Comunidades Autómomas. El reparto de competencias entre el Estado y las Comunidades, reflejado en los artículos 148 y 149 del texto constitucional, deja en manos de los emergentes poderes autonómicos cuestiones tradicionalmente atribuidas a los municipios. Así, destacadamente, la regulación del urbanismo, las ferias o en general el comercio. Los Estatutos de Autonomía, en un segundo nivel, se ocupan de definir el alcance de la competencia autonómica sobre cada una de estas materias, y suelen hacerlo con vocación de apurar el papel de la Comunidad sobre las mismas, sin pensar en el espacio propio de los entes locales a la hora de su ejecución. Es cierto que el artículo 2 de la Ley de Bases de Régimen Local ordena a los legisladores autonómicos, así como al legislador estatal, la atribución a los municipios de competencias que garanticen su derecho a intervenir cuantos asuntos afecten directamente al círculo de sus intereses ${ }^{22}$, pero también lo es que los mecanismos diseñados hasta el momento para velar por la realización de este mandato legal, reforzado sólo en tanto se deriva del principio constitucional de autonomía local, han sido insuficientes. La idea de la «garantía institucional» de la autonomía local no ha servido, como ya hemos puesto de manifiesto en otro lugar ${ }^{23}$, para evitar el vaciamiento del poder de municipios y diputaciones, y la nueva herramienta de defensa ante el Tribunal Constitucional deja muchas incógnitas pendientes de resolver que sólo futuras Sentencias estimatorias de conflictos en defensa de la autonomía local podrán despejar ${ }^{24}$.

20 Vid. De la Quadra-Salcedo, Corporaciones locales y actividad económica, Marcial Pons, Madrid, 2000.

${ }^{21}$ Vid. Sosa WAGNer, «Comentario a la desafortunada sentencia del Tribunal Supremo de 6 de septiembre de 1989», Revista del Poder Judicial, núm. 19. 1990.

22 Vid. Rivero Ysern, Manual de Derecho local, Civitas, Madrid, 1999.

${ }^{23}$ Vid. Rivero Ortega, «Autonomía local y competencias: Castilla y León en el escenario previo al Pacto Local, Revista de Estudios de la Administración Local núm. 286-287, Mayo-Diciembre 2001.

${ }^{24}$ Vid. INAP, Defensa de la autonomía local ante el Tribunal Constitucional, INAP, Madrid, 1997. 
Hay que advertir, pese a esta criticable situación en lo que se refiere al reconocimiento legislativo de competencias concretas, que la LBRL antepone a la relación de materias en las que necesariamente debe intervenir el municipio (artículo 25.2 LBRL: seguridad en lugares públicos; ordenación del tráfico; protección civil, prevención y extinción de incendios; urbanismo; patrimonio histórico-artístico; medio ambiente; abastos y consumo; salubridad pública; atención primaria a la salud; cementerios y servicios funerarios; servicios sociales; agua y alumbrado público, limpieza viaria y tratamiento de residuos; transporte público de viajeros; actividades o instalaciones culturales o deportivas; enseñanza) una cláusula general habilitadora de la iniciativa municipal cuyas virtualidades aún no han sido quizás del todo exploradas: «El Municipio, para la gestión de sus intereses y en el ámbito de sus competencias, puede promover toda clase de actividades y prestar cuantos servicios públicos contribuyan a satisfacer las necesidades y aspiraciones de la comunidad vecinal» (artículo 25.1 LBRL). Esta cláusula podría permitir al municipio ir más allá de la literalidad de la legislación estatal y autonómica, allá donde no haya una atribución competencial expresa, pero si la Comunidad Autónoma ya se ha arrogado la competencia sobre la actividad o la titularidad del servicio poco o nada puede hacer un ente local en desarrollo del apartado 1 del artículo 25 de la Ley de Bases de Régimen Local.

¿Qué posibilidades reales de intervención les restan, en este contexto, a los entes locales? ¿Pueden los municipios seguir desarrollando una actuación económica basada fundamentalmente en la reserva de servicios y la realización de actividades en ejercicio de su iniciativa pública constitucional y legalmente reconocida? Desde nuestro punto de vista, el nuevo escenario de relaciones entre poderes públicos e iniciativa privada impone la reconsideración de las técnicas contempladas como principales en la legislación básica de régimen local, y su sustitución por otras alternativas, más adaptadas a la nueva situación. Eso sí, convenientemente reguladas.

En primer lugar, debería considerarse la utilización, como herramienta de intervención económica, de la potestad de ordenanza. La regulación de actividades por parte de los municipios, empleando su potestad de ordenanza, se ha visto también sensiblemente reducida, a pesar del cada vez mayor interés doctrinal que despierta ${ }^{25}$. Debería aprovecharse el replanteamiento que se ha producido en la doctrina de nuestro Derecho administrativo sobre la importancia de los reglamentos de los entes locales para

${ }^{25}$ Vid. Blasco, Ordenanza municipal y Ley, Marcial Pons, Madrid, 2001; Galán, La potestad normativa autónoma local, Attelier, Barcelona, 2001 
precisar en la Ley de Bases el alcance y los límites de las ordenanzas reguladoras de la actividad económica privada, porque sin duda ésta es una de las herramientas más poderosas en manos de los entes locales para desarrollar una función directiva del desarrollo económico, armonizándolo con otros intereses públicos dignos de igual o superior protección, como el ambiente o el patrimonio histórico-artístico. Es cierto que hoy el artículo 84 de la Ley de Bases de Régimen Local incluye las ordenanzas entre las técnicas de intervención económica, pero parece aconsejable una regulación ulterior de su empleo concreto.

También debería profundizarse en el tratamiento doctrinal y normativo de la actividad subvencional de los municipios. Aunque existen estudios concienzudos sobre esta institución en nuestro Derecho ${ }^{26}$, lo cierto es que se han centrado sobre todo en las subvenciones del Estado. Igualmente, las normas dictadas en desarrollo de la Ley de Procedimento Administrativo Común han sido pensadas para las subvenciones concedidas por el Estado, no para las ofrecidas por los entes locales con el fin de estimular la realización de determinadas actividades de interés económico general. Es bien sabido que el fomento permite la implicación de los sujetos privados en la realización de objetivos públicos, y sin esta participación de la iniciativa privada no es factible la promoción del desarrollo económico en nuestros días.

Por esta misma razón, otras herramientas de primer orden en la nueva intervención económica de los entes locales deberían ser el convenio y la Administración paccionada, modalidades de actuación administrativa necesitadas de una mayor regulación, al menos el el ámbito local, puesto que en algunos casos se emplean para eludir normas contenidas en la legislación de contratos de las Administraciones públicas o para no ejercitar potestades unilaterales en sectores como el urbanismo. Todas las formas de colaboración público-privado, desde las más clásicas, como la concesión o la sociedad mixta, hasta las de ingeniería jurídica compleja, vía convencional o consorcial, requieren un tratamiento normativo más profundo que el dado hasta ahora en la legislación básica, sin coartar por ello ni el margen de maniobra que corresponde al legislador autonómico en el ejercicio de la competencia que le corresponde sobre régimen local (ampliamente desarrollada en algunas Comunidades Autónomas, como Cataluña, escasamente en otras, como Castilla y León) ni cohibir tampoco la iniciativa local en cuanto a la elección final de la fórmula de colaboración a adoptar en cada caso.

\footnotetext{
${ }^{26}$ Vid. Fernández-Farreres, La subvención. Concepto y régimen jurídico, IEF,1983 Madrid. Díaz LEMA, Subvenciones y Crédito Oficial en España, IEF, Madrid, 1985.
} 
El ejercicio efectivo de sus facultades de iniciativa económica y de prestación más efectiva de servicios y realización de actividades aconseja poner a disposición de los entes locales por vía normativa una definición más actual y completa del instrumental a su servicio y de las garantías jurídicas que deben rodear su utilización. Falta hoy, pensamos, salvo en alguna Comunidad Autónoma que la ha desarrollado por su cuenta, una norma que cumpla las funciones en su momento asumidas por el Reglamento de Servicios de las Corporaciones Locales de 1955. Es preciso un texto normativo nuevo, o una reforma puntual de la Ley de Bases de Régimen Local, que detalle las distintas técnicas de intervención económica y las opciones institucionales básicas para la prestación de servicios y la realización de actividades. No es preciso un texto tan pormenorizado como el Reglamento de Servicios, cuya vigencia también se hace conveniente replantear, pero sí tal vez una solución intermedia, y puesta al día, entre el contenido básico de los actuales artículos 84 a 87 de la Ley de Bases de Régimen Local y su desarrollo en el Texto Refundido de Disposiciones vigentes en materia de Régimen Local y el Reglamento de Servicios de las Corporaciones Locales de 1955.

La apreciación de la necesidad de reformas se ve confirmada tras la lectura del Proyecto de Ley de Medidas para la Modernización del Gobierno Local, proyecto que, junto a otras reformas de mayor calado, plantea cambios en «la insuficiente y superada regulación de las formas de gestión de los servicios públicos locales en la legislación básica estatal» (Exposición de Motivos del Proyecto). En este sentido, se propone una nueva definición de los servicios públicos locales en el artículo 85.1 de la Ley de Bases de Régimen Local («son servicios públicos locales los que presten las entidades locales en el ámbito de sus competencias») y se complementan los modos de gestión con el añadido de la Entidad pública empresarial local, forma de personificación a imagen y semejanza de la homónima contemplada en la Ley de Organización y Funcionamiento de la Administración General del Estado, Ley 6/1997, de 14 de abril.

En la nueva redacción propuesta del artículo 85 también se modifican las formas de gestión indirecta de los servicios públicos, optándose por una remisión al artículo 156 del Texto Refundido de la Ley de Contratos de las Administraciones Públicas, Real Decreto Legislativo 2/2000, de 16 de junio. Al remitirse a esta norma se eliminarían las referencias expresas al arrendamiento y la cooperativa como modalidades indirectas de gestión de servicios públicos locales, lo que supondría una reducción del abanico de posibles opciones a disposición de la entidad local. Claro que no se eliminan modalidades más frecuentes de prestación indirecta, pero tampoco se 
ensaya una redefinición o ampliación de las existentes. En este mismo tono normalizador restrictivo, se extiende la prohibición del actual artículo 85.2 in fine, en cuanto a los servicios que impliquen ejercicio de autoridad, más allá de la prestación indirecta, alcanzando también el límite a la opción de la sociedad mercantil de capital social exclusivamente local.

Las medidas «modernizadoras» que se introducen en materia de gestión de servicios se reducirían en el proyecto a la introducción de la nueva figura de Entidad Pública Empresarial Local, y las previsiones ampliadoras en materia de mancomunidades (permitidas entre municipios de distintas Comunidades Autónomas) y consorcios (empleables según el proyecto para la gestión de servicios en el marco de convenios de cooperación transfronteriza). Estas previsiones referidas a mancomunidades y consorcios parecen más pensadas para resolver problemas muy concretos planteados por la interpretación restrictiva de la anterior normativa que para ofrecer una respuesta a los verdaderos desafíos de la Administración local en el siglo XXI.

El proyecto de Ley, que acierta en la localización del problema de las formas de gestión, no ofrece a nuestro juicio una respuesta suficiente ni realmente adaptada a las nuevas demandas de herramientas de gestión por parte de las Corporaciones locales. La regulación en los propuestos artículos 85 bis y 85 ter presentan, más bien, la clásica aproximación uniformizadora del régimen de la Administración local y la Administración General del Estado, un error recurrente por desgracia en la evolución del Derecho local español, origen de un sinfín de inconvenientes en el respeto del principio de legalidad por las Administraciones locales.

Así, el artículo 85bis propuesto por el proyecto asimila el régimen de los organismos públicos locales y las entidades públicas empresariales locales al de sus homónimos en la LOFAGE, con numerosas especialidades organizativas. Se afecta así tanto la potestad organizatoria de la entidad local como la competencia legislativa autonómica sobre este ámbito. En cuanto a las sociedades mercantiles locales, el artículo 85ter se muestra más escueto, sin ampliar las posibilidades de empleo de esta figura en la realización de las tareas de los entes locales.

Lejos de abrir nuevas posibilidades de actuación económica de los entes locales, el proyecto de Ley optar por mantener el esquema existente, aproximando las formas de gestión a las propias de la Administración del Estado. 


\section{LA ACTIVIDAD ECONÓMICA DE LOS ENTES LOCALES EN COLABORACIÓN CON OTROS ENTES PÚBLICOS, LA COLABORACIÓN PÚBLICO-PRIVADO, Y LA REGULACIÓN DE LA OPCIÓN INSTITUCIONAL}

A nuestro juicio, la nueva regulación de la actividad económica de los entes locales debería tener en cuenta las dos grandes dificultades de la realización de las funciones económicas y de prestación de servicios de las Corporaciones locales, que son, de acuerdo con las tesis expuestas en este trabajo, tanto la atribución de competencias a las Comunidades Autónomas que se solapan con tareas tradicionalmente encomendadas a los entes locales, lo que obliga a tomar en cuenta las decisiones de varios niveles sin disponer de total autonomía en el desarrollo de estas labores, como el replanteamiento del papel de los poderes públicos respecto de la economía, y el nuevo reparto de funciones entre éstos y la iniciativa privada, asociado a la crisis financiera constante de los municipios y diputaciones, que dificultaría asumir nuevas tareas o plantear nuevos proyectos al carecer de capacidad presupuestaria suficiente

Ante esta situación, se hace imprescindible fortalecer la colaboración como mecanismo alternativo de realización de las funciones económicas de los entes locales, y para ello, es preciso afinar el tratamiento normativo de la creación de organizaciones compartidas con el fin de promocionar la creación de riqueza. Los consorcios locales, las sociedades mixtas de entes públicos y la herramienta del convenio entre sujetos públicos y sujetos privados requieren una actualización profunda porque se convierten, en las circunstancias anteriormente expuestas, en instrumentos claves para poder realizar la función pública de promover el desarrollo económico mediante el impulso de proyectos adaptados a las necesidades de cada espacio local. La legislación de Bases de Régimen Local contempla estas técnicas, pero lo hace de forma escueta, sin desarrolllarlas lo suficiente como para que se puedan resolver los principales problemas que plantea su empleo. No se enfrenta del todo a la necesidad de una colaboración mucho mayor entre entidades locales para la realización de tareas cuyo cumplimiento exige aunar cada vez más esfuerzos.

Concluyendo: una revisión de la Ley de Bases de Régimen Local en lo relativo a la intervención económica de los entes locales parece a estas alturas aconsejable. La asunción de la competencia de régimen local por la práctica totalidad de las Comunidades Autónomas, que hace previsible el futuro dictado de leyes autonómicas más completas sobre esta materia, también aboga por una revisión de contenidos tanto en la Ley de Bases 
como en el Texto Refundido. Y si pueden hacerse estas consideraciones respecto de la Ley de Bases y el Texto Refundido, normas post-constitucionales de mediados de los años ochenta, con mayor razón pueden hacerse respecto de lo que pueda permanecer vigente del Reglamento de Servicios de las Corporaciones Locales, una norma de gran rigor técnico e impecable como respuesta jurídica a la realidad de la intervención económica de los entes locales durante casi cincuenta años, probablemente demasiados para una cuestión como ésta, que el merecidamente recordado Profesor Doctor Sebastián MARTín-RetortiLlo consideró de gran alcance ${ }^{27}$.

\footnotetext{
27 Vid. Sebastián MARTíN-ReToRTILLo, «La actuación de las Corporaciones locales (Acotaciones al proyecto de Ley de regulación de las Bases del Régimen local)», Revista Española de Derecho Administrativo núm. 42, Abril-Junio 1984.
} 